\title{
Effect of Transmission Parameters on PAPR of Universal Filter Multicarrier Modulation Systems
}

\author{
Pooja Rani, Silki Baghla and Himanshu Monga* \\ Department of ECE, JCDM College of Engineering, India \\ *Corresponding author: Himanshu Monga, Department of ECE, Director of JCDM College of Engineering, India

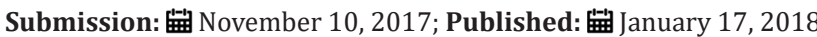

\begin{abstract}
One of the main objectives of multicarrier modulation is to provide multiple accesses for wireless communication systems with higher data rates while having minimum out of band radiation, high spectral efficiency and less complexity. Orthogonal frequency division multiplexing (OFDM), Universal filter multicarrier (UFMC), Filter bank multicarrier (FBMC) and Generalized frequency division multiplexing (GFDM) modulation techniques have been developed to support fourth generation and beyond 4G wireless systems. Demand of high data rate in fourth generation wireless communication systems has been fulfilled by OFDM techniques but it suffers from the limitation of less spectral efficiency and high PAPR (Peak to average power ratio). Thus to support next generation wireless systems other waveform models are getting attention. Among the techniques available, UFMC seems to be attractive due to high spectral efficiency and less complexity. It has not explored much so in this paper, performance of UFMC have been evaluated with different design factors such as number of sub bands, FFT (Fast Fourier Transform)size, filter characteristics and modulation under the light of PAPR.

Keywords: OFDM; GFD; UFMC; FBMC; FFT; PAPR
\end{abstract}

\section{Introduction}

To support next generation wireless communication system with higher data rates, less interference and lower PAPR a new waveform candidate is required. Various new modulation schemes like UFMC, FBMC, OFDM and GFDM have been introduced and studied in different ways [1,2]. OFDM is one of the most popular and accepted modulation techniques in many communication systems. In OFDM, to increase robustness against Inter-Symbol Interference (ISI) caused by multipath propagation on the radio channel the total symbol duration is further increased by a adding a Cyclic Prefix (CP). A CP is a copy of the tail of a symbol placed at its beginning. However, the insertion of cyclic prefix degrades its spectral efficiency and makes it less reliable for next generation wireless communication. On the other hand, FBMC improves spectral efficiency of system by using an inverse fast Fourier transform/fast Fourier transform (IFFT/FFT) based filter bank, and staggered OQAM (Orthogonal Quardrature Amplitude Modulation) symbols, real symbols at twice the symbol rate of FBMC/QAM, are loaded on the subcarriers. Hence, FBMC/OQAM has a higher spectral efficiency but at the cost of increased system complexity. UFMC seems to be attractive technique as it combines the advantages of OFDM and FBMC [3]. It provides better sub carrier separation like FBMC and less complexity like OFDM. Filter bank multicarrier (FBMC) filters the signal on per subcarrier basis [4] while orthogonal frequency division multiplexing (OFDM) filters the signal on single shot [5]. UFMC (Universal filter multicarrier) can be seen as a compromise between OFDM and FBMC, it groups subcarriers to sub-bands, which are then filtered individually as compared with single subcarrier or the complete band. In each sub band, filter parameters and number of carrier per remains same to prevents aliasing [6]. But all these multi carrier modulation schemes suffer from the problem of higher peak to average power ratio (PAPR). In this paper we have evaluated the effect of various design parameters like filter length, FFT size, modulation order and number of sub bands on the peak to average power ratio of Universal filter multi carrier modulation technique.

The remainder of this paper is organized as follows: In section II, the considered UFMC system model is presented. Section III is devoted to the high Peak to average power ratio. In Section VI reports simulation setup, including the performance evaluation of UFMC in light of PAPR with different design factors. Finally, conclusions are drawn in Section V.

\section{UFMC System}

UFMC system was developed based on the principle of frequency division multiplexing (FDM) in which we divide the input data stream into several lower rate sub-streams [7]. The block diagram of a system employing UFMC is depicted in Figure 1 as shown in figure, the data stream is divided into sub-streams, 
and then a pulse shaping filter with smooth edges is used on each resource block in time domain that leads to substantial reduction in out-of-band leakage in frequency domain. In this way, it minimizes the harmful interference from adjacent sub channels of the neighboring resource block [8].

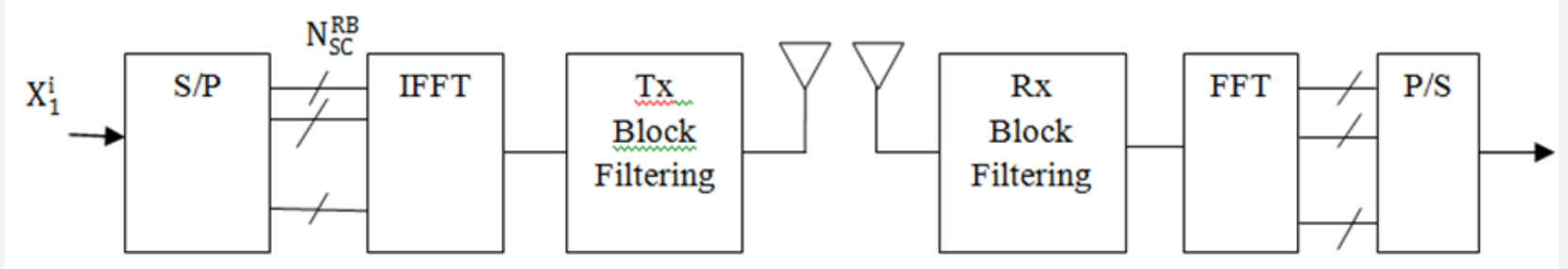

Figure 1: UFMC system model.

As shown in Figure 1, the generated output can be given as:

$$
x=\sum_{p=1}^{N_{R B}} F_{p} V_{p} X_{k}^{i}
$$

$\mathrm{V}_{\mathrm{p}}$, being of dimension $N_{S C} \times N_{S C}^{R R}$ includes the relevant columns of the inverse Fourier matrix according to the respective sub-band position within the overall available frequency range, Fp is a Toeplitz matrix with dimension $\left(N_{S C}+N_{\text {Filter }}-1\right) \times N$, composed of the filter impulse response, enabling the convolution. The signal can be rewritten without the summation by the following definitions:

$$
\begin{gathered}
\bar{F}=\left[F_{1}, F_{2}, F_{3}, \ldots \ldots . F_{N_{R B}}\right] \\
\overline{V_{p}}=\operatorname{diag}\left(V_{1}, V_{2}, V_{3}, \ldots . . V_{N_{R B}}\right) \\
\overline{X_{k}^{i}}=\left[X_{k, 1}^{i^{T}}, X_{k, 2}^{i^{T}}, X_{k, 3}^{i^{T}}, \ldots \ldots X_{k, N_{R B}}^{i^{T}}\right]^{T} \\
x=\bar{F} V_{p} X_{k}^{i}
\end{gathered}
$$

The block-wise filtering provides flexibility to the system and may be used to avoid the main FBMC drawbacks. UFMC supports short bursts data transmission, as well as operation in fragmented bands. The filter provides protection against inter-symbol interference (ISI), as well as robustness for supporting multiple access users which are not perfectly time-aligned. Due to the possibility to reduce guard bands, and to avoid using a CP, UFMC is spectrally more efficient than CP-OFDM. The receiver processing can still be similar to CP-OFDM, single-tap per-subcarrier frequency domain equalizers can be used which equalize the joint impact of the radio channel and the respective sub band-filter [9]. This leads to similar complexity order as CP-OFDM. So, it is thereby clear that UFMC provides advantages of both OFDM and FBMC system [10].

\section{Peak to Average Power Ratio}

The PAPR is the relation between the maximum power of a sample in a given transmit symbol divided by the average power of that symbol. PAPR occurs when in a multicarrier system the different sub-carriers are out of phase with each other [11]. At each instant they are different with respect to each other at different phase values. When all the points achieve the maximum value simultaneously, this will cause the output envelope to suddenly increase which causes a 'peak' in the output. There are a large number of independently modulated subcarriers in multicarrier system, and when they are added up coherently for transmission purpose give a large peak value which is very large as compared to average value of the sample. The ratio of the peak to average power value is termed as Peak-to-Average Power Ratio and it can be given as:

$$
\text { PAPR }=\left(\frac{\max \left\{|x[n]|^{2}\right\}}{E\left\{|x[n]|^{2}\right\}}\right)
$$

Where, is the amplitude of $\mathrm{x}[\mathrm{n}]$ and E denote the expectation of the signal.

PAPR in $\mathrm{dB}$ can be written as:

$$
\operatorname{PAPR}(\mathrm{dB})=10 \log _{10}(\mathrm{PAPR})
$$

This higher PAPR causes saturation in power amplifier which produces inter modulation products among sub bands and also increases out of band radiation [12].

\section{Different Factors Effecting PAPR in UFMC}

There are various parameters which can effect UFMC signal generation [13]. In this section we have given a brief of all these design parameters, which we should take in account while designing an UFMC system model.

\section{Filter characteristics}

For the choice of the filter characteristics many alternatives are available. In this experimental work we have used Dolph-chebyshev filter and evaluated effect of different filter lengths on PAPR of UFMC. For a given type of sub band filter, the filter length is a key parameter affecting the system performance in different ways. A longer filter not only leads to lower out of band emission, but also results in better frequency localization, and makes the system more robust to synchronization errors and multipath fading channels.

However, a longer filter also causes several drawbacks including more frequency selective filter response along subcarriers within one sub band and larger overhead, reducing transmission efficiency in the time domain [14]. 


\section{Modulation type and its orders}

There are various modulation schemes which can be use for UFMC system model such as BPSK, QAM, and QPSK. For this evaluation we have used QAM modulation and studied effect of different modulation orders such as 4QAM, 16QAM, 64QAM and 256QAM. Modulation order of the system plays an important role on system performance and its PAPR value also get affected by this $[15,16]$.

\section{FFT size}

The third factor taken in account is FFT size of the transmitter. We have evaluated PAPR of UFMC by varying FFT size. For example: 256-point FFT, 512-point FFT, 1024-point FFT and 2048-point FFT [16].

\section{Number of sub bands}

The sub band size is also having a great impact on signal generation of UFMC model. Number of sub bands, FFT size, modulation order and filter length are those key factors which can change signal generation in a large way. In this paper we are evaluating impact of these factors on system performance.

\section{Simulation Setup}

In this section we have evaluated that how different design factors of UFMC model affects its PAPR value. Different design parameters for simulation setup are summarized in Table 1.

Table 1: Simulation parameters for considered UFMC system.

\begin{tabular}{|c|c|}
\hline Parameter & Value \\
\hline Filter Characteristics & $\begin{array}{c}\text { Dolph-chebyshev filter with filter } \\
\text { length- 43, 63, 83 }\end{array}$ \\
\hline Modulation Order & QAM- 4,64,256 \\
\hline FFT Size & FFT point- 256, 512, 1024, 2048 \\
\hline Number of sub bands & $20,30,40$ \\
\hline
\end{tabular}

Table 2: PAPR values with different filter length.

\begin{tabular}{|c|c|c|c|c|}
\hline $\begin{array}{c}\text { Number of } \\
\text { Sub Bands }\end{array}$ & $\begin{array}{c}\text { Filter } \\
\text { Length }\end{array}$ & $\begin{array}{c}\text { Modulation } \\
\text { Order }\end{array}$ & FFT Size & PAPR \\
\hline 20 & 43 & 256 & 512 & 22.9060 \\
\hline 20 & 63 & 256 & 512 & 21.8020 \\
\hline 20 & 83 & 256 & 512 & 20.2872 \\
\hline
\end{tabular}

Table 2 gives different values of filter length and the corresponding values of PAPR are also given. Figure 2 is showing the effect of different filter length on PAPR of system, here we are using Dolph-chebyshev filter for realization of the system. It is clear from table and figure that as we increase the filter length it reduces the PAPR and vice-versa.

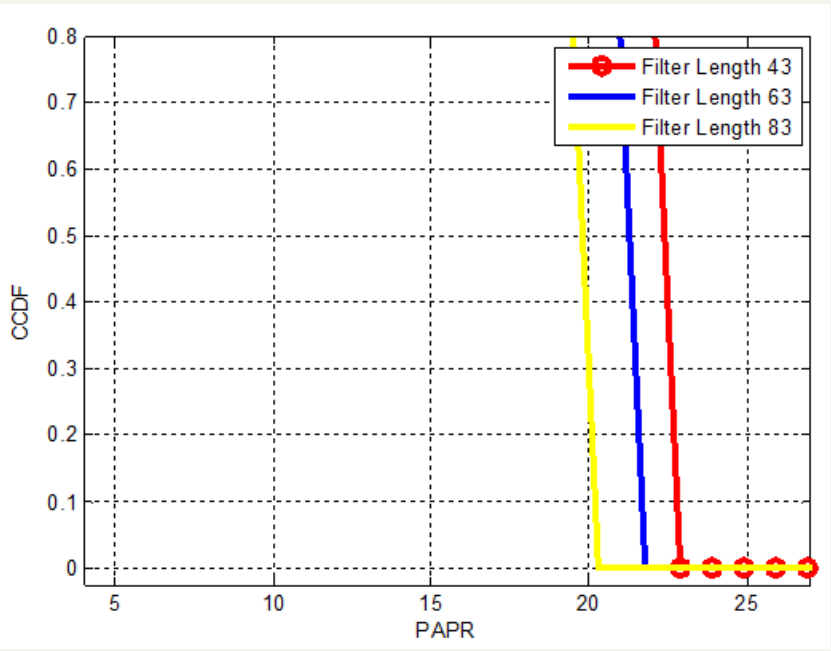

Figure 2: Effect of filter length on PAPR of UFMC.

We can say that a higher filter length can achieve a better PAPR performance but it also makes the filter more frequency selective which in turn reduces it bandwidth. Table 3 is showing different values of PAPR with change in modulation order of the system and corresponding values are shown graphically in Figure 3. From this it is clear that UFMC is having minimum value of PAPR at moderate modulation order and peak to average power ratio is higher at both low and high modulation orders, it keeps on increasing as the modulation order is increased. The next factor of our observation is that how FFT size of the transmitter affects the PAPR of the system. Table 4 is showing values of PAPR corresponding to the FFT size of the transmitter and Graphical representation is given in Figure 4.

Table 3: PAPR values with different modulation orders.

\begin{tabular}{|c|c|c|c|c|}
\hline $\begin{array}{c}\text { Number of } \\
\text { Sub Bands }\end{array}$ & $\begin{array}{c}\text { Filter } \\
\text { Length }\end{array}$ & $\begin{array}{c}\text { Modulation } \\
\text { Order }\end{array}$ & FFT size & PAPR \\
\hline 20 & 43 & 4 & 512 & 21.7315 \\
\hline 20 & 43 & 64 & 512 & 20.7673 \\
\hline 20 & 43 & 256 & 512 & 22.9060 \\
\hline
\end{tabular}

Table 4: PAPR values with different FFT size.

\begin{tabular}{|c|c|c|c|c|}
\hline $\begin{array}{c}\text { Number of } \\
\text { Sub Bands }\end{array}$ & $\begin{array}{c}\text { Filter } \\
\text { Length }\end{array}$ & FFT Size & $\begin{array}{c}\text { Modulation } \\
\text { Order }\end{array}$ & PAPR \\
\hline 20 & 83 & 256 & 256 & 17.8552 \\
\hline 20 & 83 & 512 & 256 & 20.2872 \\
\hline 20 & 83 & 1024 & 256 & 21.8360 \\
\hline 20 & 83 & 2048 & 256 & 20.2337 \\
\hline
\end{tabular}




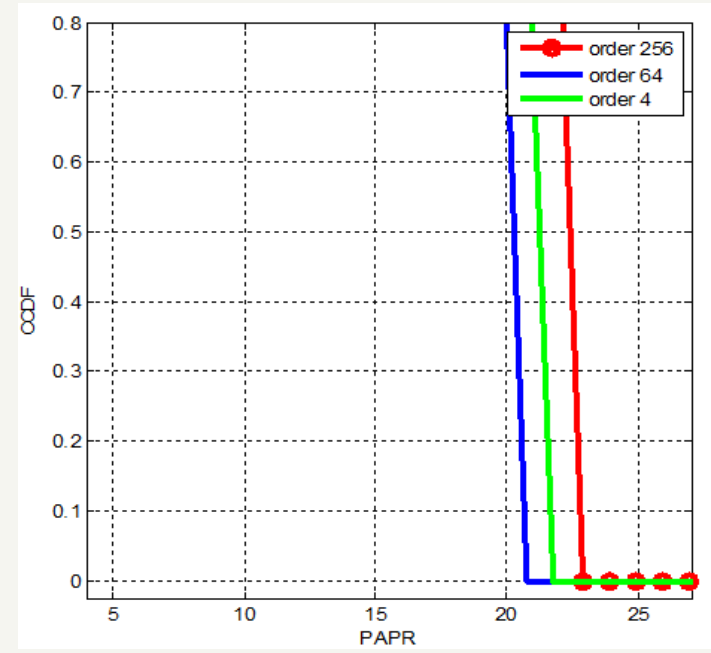

Figure 3: Effect of modulation order on PAPR of UFMC.

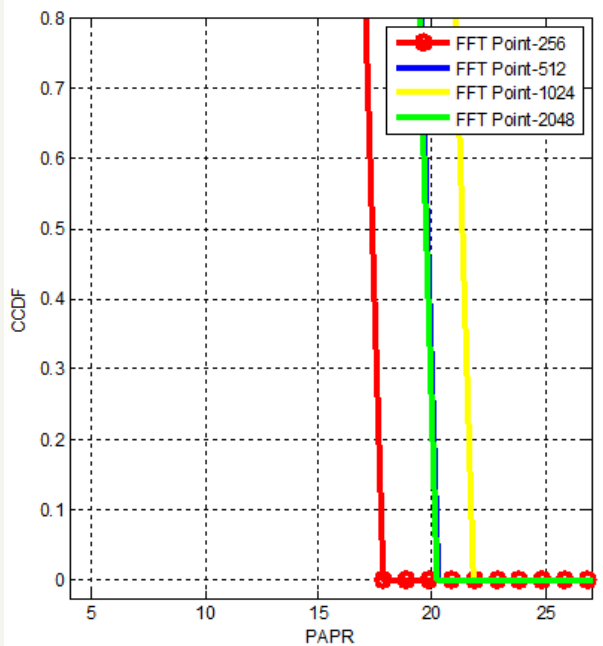

Figure 4: Effect of FFT Size on PAPR of UFMC.

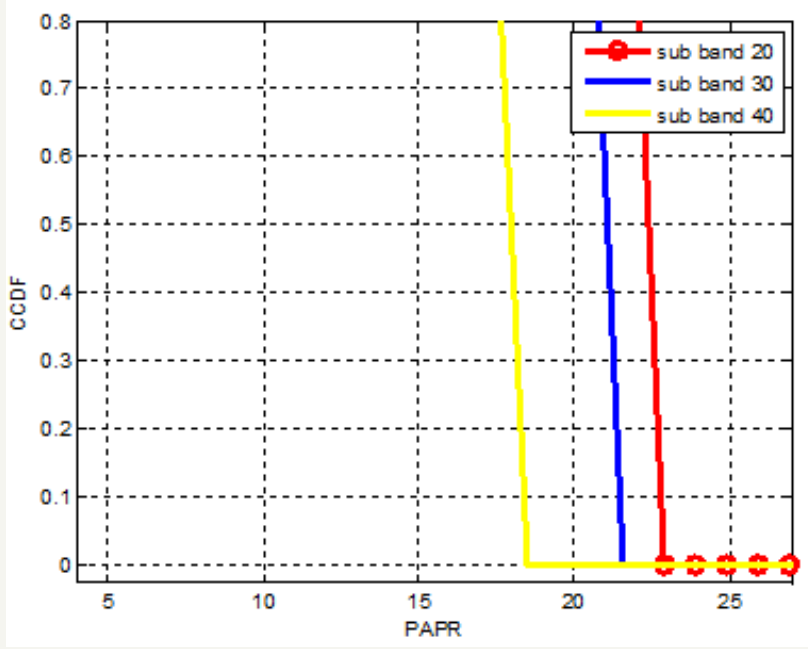

Figure 5: Effect of Number of Sub bands on PAPR of UFMC.

\section{Conclusion}

In this paper we have studied that how different design factors of an UFMC model can affect its performance because a higher PAPR value put a great impact on system performance and degrades it. So, depending on these experimental outcomes we can design such an UFMC model which give lower value of PAPR. This evaluation also shows that although UFMC modulation is having advantages of both FBMC and OFDM but it is having limitation of higher peak to average power ratio like other two modulation schemes. So, in future we can focus on some PAPR reduction techniques to overcome this shortcoming of the UFMC modulation to make it more reliable and efficient for next generation wireless communication.

\section{References}

1. Rohde, Schwarz (2016) Application Note "5G waveform candidates".

2. 5GNOW (2013) D3.1: 5G waveform candidate selection. Tech Rep.

3. Schaich F, Wild T (2014) Waveform contenders for 5G-OFDM vs. FBMC vs. UFMC. ISCCSP pp. 457-460.

4. Farhang-Boroujeny B (2011) OFDM versus filter bank multicarrier. IEEE Signal Process Mag 28(3): 92-112.

5. Bingham John (1990) Multi carrier modulation for data transmission: An idea whose time has come. IEEE Commun Mag 28(5): 5-14.

6. Vakilian V, Wild T, Schaich F, Ten Brink S, Frigon JF (2013) Universalfiltered multi-carrier technique for wireless systems beyond LTE. IEEE Globecom Workshops pp. 223-228.

7. Wunder G, Jung P, Kasparick M, Wild T, Schaich F, et al. (2014) 5GNOW: non-orthogonal, asynchronous waveforms for future mobile applications. IEEE Commun Mag 52(2): 97-105.

8. Chen Y, Schaich F, Wild T (2014) Multiple access and waveforms for 5G: IDMA and universal filtered multi-carrier. IEEE $79^{\text {th }}$ VTC Spring pp. 1-5.

9. Wild T, Schaich F, Chen Y (2014) 5G Air interface design based on universal filtered (UF-)OFDM. $19^{\text {th }}$ International Conference on Digital Signal Processing pp. 699-704.

10. An C, Kim B, Ryu HG (2016) Waveform comparison and nonlinearity sensitivities of FBMC, UFMC and W-OFDM systems. $8^{\text {th }}$ International Conference on Networks \& Communications, pp. 83- 90.

11. Gangwar A, Bhardwaj M (2012) An overview: peak to average power ratio in OFDM system \&its Effect. IJCOTS 1(2): 22-25.

12. Tellado J, Cioffi JM (1999) PAR reduction in multicarrier transmission system. Stanford University, USA.

13. Zhang L, Ijaz A, Xiao P, Ali Imran M, Tafazolli R (2016) MU-UFMC system performance analysis and optimal filter length and zero padding length design.

14. Elmaroud B, Faqihi A, Abbad M, Aboutajdine D (2014) On the impact of prototype filter length on the PAPR reduction of FBMC signals. IJET 6(4): 1951-1960.

15. Chandra G, Dinesh KS, Anuj S (2013) Examine the impact of modulation order and sub-bands on PAPR reduction techniques using various modulator in $802.11 \mathrm{a} / \mathrm{b} / \mathrm{g}$. International Journal of Computer Applications (0975 - 8887) 69(6): 49-56.

16. Abdullah MFL, Ghanim MF, Yonis AZ (2013) Effects of FFT size on PAPR of MC-CDMA system. IEEE $9^{\text {th }}$ International Colloquium on Signal Processing and its Applications. 